\title{
Escala Multidimensional de Satisfação de Vida para Crianças: Revisão e Normas
}

\author{
Cyntia Mendes de Oliveira1 ${ }^{1}$ Euclides José de Mendonça Filho, \\ Aline Riboli Marasca, Denise Ruschel Bandeira, Claudia Hofheinz Giacomoni \\ Universidade Federal do Rio Grande do Sul, Porto Alegre-RS, Brasil
}

\section{RESUMO}

A Escala Multidimensional de Satisfação de Vida para Crianças (EMSVC) avalia a satisfação de vida em crianças entre sete e 12 anos de idade por meio de 50 itens distribuídos em seis dimensões. O objetivo deste estudo foi revisar as propriedades psicométricas da EMSVC e criar normas para a sua utilização. A amostra foi composta por 1181 crianças de sete a 13 anos de idade $(M=10,43 ; D P=1,78)$ de dados oriundos de pesquisas realizadas no Rio Grande do Sul. Foi conduzida uma análise fatorial confirmatória para avaliar a qualidade de ajuste do modelo. A fidedignidade foi estimada por meio do alfa de Cronbach e ômega de McDonald. Os resultados indicaram que as alterações favoreceram a obtenção de bons índices de ajuste do modelo após a exclusão de 18 itens e retirada da dimensão Não Violência. Houve incremento na consistência interna das subescalas. Este estudo fornece normas percentílicas por sexo e ano escolar para uso clínico e em pesquisa.

Palavras-chave: avaliação psicológica; crianças; satisfação de vida; validação de teste.

\section{ABSTRACT - Multidimensional Life Satisfaction Scale for Children: Revision and Standards}

The Multidimensional Life Satisfaction Scale for Children (MLSS-C) assesses life satisfaction in children aged between seven and 12 years, through 50 items distributed among six dimensions. The aim of this study was to revise the psychometric properties of the MLSS-C and create standards for its use. The sample was composed of data from 1181 children aged seven to 13 years $(M=10.43$; $S D=1.78$ ) from studies conducted in the state of Rio Grande do Sul. Confirmatory factor analysis (CFA) was performed to evaluate the quality of the model fit. The reliability was estimated using Cronbach's alpha and McDonald's omega. Results showed good model fit after the exclusion of 18 items, the Non-Violence dimension was also removed. Results indicated an improvement of its indexes after psychometric revision and an increase in the internal consistency of the subscales. Percentile standards according to participants' sex and school year are provided for clinical and research use. Keywords: children; life satisfaction; psychological assessment; test validity.

\section{RESUMEN - Escala Multidimensional de Satisfacción con la Vida para Niños: Revisión y Normas}

La Escala Multidimensional de Satisfacción con la Vida para Niños (EMSVC) evalúa la satisfacción con la vida de niños entre siete y 12 años de edad a través de 50 ítems distribuidos en seis dimensiones. El objetivo de este estudio fue revisar las propiedades psicométricas de la EMSVC y crear normas para la utilización de dicha escala. La muestra fue compuesta por 1181 niños entre siete y 13 años de edad $(M=10,43 ; D S=1,78)$, con datos provenientes de investigaciones realizadas en la província de Rio Grande do Sul. Se realizó un análisis factorial confirmatorio para evaluar la calidad de ajuste del modelo. La fiabilidad fue estimada por el alfa de Cronbach y por el omega de McDonald. Los resultados exhiben que las alteraciones favorecen la obtención de buenos índices de ajustes del modelo tras excluir 18 ítems y retirar la dimensión No-Violencia. Se ha producido un incremento en la consistencia interna de las subescalas. Esta investigación proporciona normas porcentuales por sexo y año escolar para uso clínico y en investigación.

Palabras clave: evaluación psicológica; niños; satisfacción con la vida; validez de test.

Um dos construtos centrais na literatura da Psicologia Positiva é a satisfação de vida. O conceito atrai a atenção de pesquisadores e profissionais de diferentes campos de atuação, por propor um indicador da qualidade de vida da população a partir de critérios subjetivos (Diener et al., 2017). A satisfação de vida é uma das dimensões do bem-estar subjetivo, reconhecida como uma avaliação cognitiva sobre a vida de maneira geral e nas suas diferentes áreas (Diener, 1984; Gilman \& Huebner, 2006). Seu acesso pode ser realizado a partir de uma medida global ou de maneira multidimensional, com a discriminação de domínios específicos da vida. 
Tais medidas fornecem informações para programas de intervenções que buscam aumentar a satisfação de vida global em diversos contextos.

Os instrumentos multidimensionais possibilitam o acesso aos domínios específicos da satisfação de vida, os quais estão intimamente ligados às experiências concretas. Além disso, oferecem informações diferenciadas relacionadas a cada domínio (Suldo, Huebner, Friedrich, \& Gilman, 2009). Os modelos multidimensionais buscam delinear as áreas mais relevantes para o indivíduo avaliar a qualidade de sua vida, contudo, elas podem diferir conforme a fase do desenvolvimento (Suldo, Frank, Chappel, Albers, \& Bateman, 2014). Para os períodos da infância e adolescência, o modelo multidimensional concebido por Huebner (1994), que embasa a Escala Multidimensional de Satisfação de Vida de Estudantes, engloba os domínios Família, Escola, Amizade, Self e Ambiente no qual a criança vive, sugerindo que, assim como em adultos, a avaliação por dimensões também é relevante nessas faixas etárias.

No Brasil, o modelo proposto por Huebner (1994) foi revisto e ampliado por Giacomoni e Hutz (2008) que, levando em consideração características do contexto, criaram a Escala Multidimensional de Satisfação de Vida para Crianças (EMSVC). A EMSVC é composta por 50 itens em seis dimensões. Entre os fatores que compõem a escala, os domínios Self, Família, Amizade e Escola assemelham-se ao modelo anterior. Foram adicionados os fatores Self Comparado e Não Violência, considerados importantes para as crianças brasileiras. Inicialmente, o modelo proposto por Giacomoni (2002) incluiu a dimensão Não Violência a partir dos achados de um estudo qualitativo em que as crianças foram entrevistadas sobre o que elas pensavam a respeito da felicidade. $\mathrm{Na}$ análise de conteúdo, a categoria de resposta Não Violência surgiu em três de 13 questões e foi considerada relevante pela autora. Entretanto, a categoria apareceu em menor frequência no relato das crianças, quando comparado com as outras categorias. Além disso, a subescala foi composta por apenas quatro itens e apresentou baixa fidedignidade $(\alpha=0,66)$.

A respeito do fator Self Comparado, este surgiu a partir do agrupamento de itens relacionados ao lazer, à amizade e à satisfação de desejos e afetos. A fidedignidade da subescala foi considerada satisfatória $(\alpha=0,86)$.

O domínio Selfé composto por 10 itens que descrevem o sujeito com características positivas, como autoestima, bom-humor, capacidade de demonstrar afeto e de relacionar-se. Self Comparado (oito itens) tem conteúdos referentes às avaliações comparativas com seus pares em relação ao lazer, à amizade e à satisfação de desejos e afetos. O fator Família (11 itens) descreve um ambiente familiar saudável permeado por afetos positivos e indicações de satisfação quanto à diversão. O fator Amizade (10 itens) refere-se às relações com pares e a satisfação desses relacionamentos, além de itens relacionados ao lazer, diversão e apoio social. O domínio Escola (sete itens) descreve a importância do ambiente escolar e dos relacionamentos interpessoais nesse espaço. Por último, o fator Não Violência (quatro itens) está relacionado aos comportamentos agressivos.

No estudo que deu origem à EMSVC (Giacomoni \& Hutz, 2008), os autores apresentaram os passos da construção da escala e um estudo de validação. As propriedades psicométricas da EMSVC foram investigadas por meio de uma análise fatorial exploratória (AFE) em uma amostra de 661 estudantes entre sete e 12 anos, indicando evidência relacionada à estrutura interna e variáveis critério. $\mathrm{O}$ alfa de Cronbach obtido para a escala total foi de 0,93 e as subescalas apresentaram confiabilidade satisfatória, variando entre 0,82 e 0,86 , com exceção da subescala Não Violência, que apresentou um valor de 0,66 , justificado pelo pequeno número de itens desse fator. Os autores não encontraram diferenças significativas entre os sexos em nenhum domínio do instrumento. Entretanto, foram observadas diferenças significativas entre as faixas etárias em cada subescala.

Desde o seu desenvolvimento, a EMSVC vem sendo empregada para a investigação da satisfação de vida de crianças em diferentes contextos, a saber, estudos com crianças com altas habilidades (França-Freitas, Del Prette, \& Del Prette, 2017), vítimas de abuso sexual intrafamiliar (Pincolini \& Hutz, 2012) e em situações de vulnerabilidade social (Dell'Aglio \& Siqueira, 2010; Poletto \& Koller, 2011). Destaca-se ainda o uso do instrumento em pesquisas realizadas em Portugal, que empregaram a escala a fim de avaliar o efeito de programas de intervenção em bem-estar subjetivo em crianças (Gonçalves, 2013; Pedro, 2017), assim como investigar a satisfação de vida infantil em contexto de institucionalização (Martins, 2012). Para viabilizar sua utilização em outro país, foi realizada a adaptação semântica em alguns itens para adequar-se à população portuguesa (Martins, 2012). Por essa razão, verifica-se o interesse de pesquisadores em colaborar com a avaliação das propriedades psicométricas da escala e com a adaptação do instrumento para uso em outros países, considerando seus resultados iniciais promissores.

Em um estudo recente (Cassoni, Marturano, Coimbra, \& Fontaine, 2017), os autores investigaram a estrutura fatorial da EMSVC com uma amostra de 379 crianças de escolas públicas do estado de São Paulo. Para isso, realizou-se uma análise fatorial confirmatória (AFC) dos 50 itens inicialmente propostos por Giacomoni \& Hutz (2008), entretanto, não se obteve ajuste adequado, evidenciando itens com baixas cargas fatoriais. A partir desses dados, sugeriu-se uma versão final com 36 itens, sendo quatro itens para a dimensão Self, sete itens para Self Comparado, dois itens para Não Violência, nove itens para Família, oito itens para Amigos e seis itens para Escola. $\mathrm{O}$ alfa da escala geral foi de 0,86 , e cinco dimensões apresentaram consistência 
interna de 0,73 a 0,88 . Contudo, a dimensão Não Violência teve um alfa de 0,50 , sendo indicada a necessidade de revisão dessa dimensão, devido sua baixa confiabilidade (Cassoni et al., 2017).

Outra proposta de redução da escala foi apresentada no estudo que tinha como objetivo investigar os efeitos de uma intervenção em educação positiva em crianças portuguesas (Pedro, 2017). Nessa versão, o instrumento foi reduzido para 28 itens, referentes às dimensões Escola (sete itens), Amizade (10 itens) e Família (11 itens). Os alfas obtidos nos momentos de pré e pós-teste para as dimensões foram de 0,70 e 0,82 para Amizade, de 0,65 e 0,84 para Família e de 0,57 e 0,86 para Escola. Em outro estudo (Bandeira, 2014), a EMSVC foi reduzida para 27 itens divididos entre quatro fatores: Self Comparado, Família, Amizade e Escola. Os alfas obtidos não foram apresentados. Apesar de não serem especificados, nos dois estudos, os procedimentos que levaram a esses resultados, verifica-se uma demanda de pesquisadores em diminuir o tamanho do instrumento.

Ainda que seja evidenciado um aumento no uso de instrumentos baseados em Psicologia Positiva no Brasil, verifica-se que medidas direcionadas às crianças ainda são incipientes, demandando maior investimento em novas escalas, assim como na avaliação da qualidade dos instrumentos disponíveis (Pires, Nunes, \& Nunes, 2015; Pureza, Kuhn, Castro, \& Lisboa, 2012). Ademais, aprimorar a qualidade das medidas fornece uma base mais segura para o desenvolvimento e avaliação de intervenções em Psicologia Positiva, área ainda pouco explorada nas pesquisas nacionais (Reppold, Gurgel, \& Schiavon, 2015). Destaca-se ainda o reconhecimento do potencial da EMSVC como um instrumento útil para a investigação de um dos componentes do bem-estar, sendo empregado por pesquisadores de diferentes regiões do país, tanto em estudos empíricos quanto com o objetivo de revisão de suas propriedades psicométricas. Visto sua importância no uso em pesquisas e na prática clínica, o objetivo deste estudo foi revisar as propriedades psicométricas e criar normas para a utilização da versão revisada da EMSVC. Além disso, é esperado que a revisão resulte em uma versão reduzida da escala, facilitando seu uso com crianças, em especial na prática em pesquisa, já que a grande quantidade de itens do instrumento original tem sido apontada como uma limitação.

\section{Método}

\section{Participantes}

A amostra que compôs o presente trabalho é resultado de três pesquisas que utilizaram a EMSVC nos últimos 10 anos. A amostra final compreendeu 1181 crianças entre sete e 13 anos de idade $(M=10,43 ; D P=1,78$ anos), sendo $50,3 \%$ do sexo masculino. Os estudantes frequentavam entre o primeiro e o sétimo ano do ensino fundamental, de escolas públicas e privadas do estado do Rio Grande do Sul.

\section{Instrumento}

A EMSVC contém 50 itens, distribuídos em seis fatores: Self (10 itens), Self Comparado (oito itens), Não Violência (quatro itens), Família (11 itens), Amizade (10 itens) e Escola (sete itens). Para responder ao instrumento, a criança lê cada frase e marca o quanto concorda em uma escala Likert de cinco pontos: (1) nem um pouco, (2) um pouco, (3) mais ou menos, (4) bastante e (5) muitíssimo. As respostas dos participantes podem compor seis subescores, além de um escore geral de satisfação de vida.

\section{Procedimentos}

A partir do levantamento acerca das publicações utilizando a EMSVC desde a sua primeira publicação (Giacomoni \& Hutz, 2008), foi feito contato com pesquisadores de grupos parceiros cujas coletas foram realizadas com crianças, a fim de solicitar autorização para utilização dos seus dados da EMSVC. Seguindo a orientação do Comitê de Ética para procedimentos no compartilhamento de dados, aqueles pesquisadores que concordaram em ceder seus dados assinaram um Termo de Compromisso de Dados. Os três bancos cedidos foram de pesquisas realizadas no estado do Rio Grande do Sul. A fim de evitar o enviesamento dos resultados, os dados provenientes dos estudos que já utilizaram partes dessa amostra não foram comparados aos dados aqui obtidos.

\section{Procedimentos de análise de dados}

Para a revisão da EMSVC, foram realizadas análises fatoriais confirmatórias. O modelo proposto por Giacomoni e Hutz (2008) foi analisado e modificações foram realizadas para alcance do ajuste do modelo aos dados. Itens com cargas fatoriais não significativas $(p>0,05)$ foram excluídos e o novo modelo foi analisado novamente. Também foi testado o modelo da EMSVC com um fator geral de segunda ordem composto pelos seis fatores da escala para se investigar a possibilidade de obtenção de um escore geral de satisfação de vida.

A matriz de correlações policóricas dos dados provenientes do instrumento foi submetida ao método de estimação Weighted Least Squares. Esse método foi escolhido porque a distribuição normal das respostas aos itens não é um pressuposto, apesar da distribuição normal das variáveis latentes serem necessárias, e por oferecer estimativas mais precisas e menos enviesadas para indicadores categóricos de nível ordinal (Flora \& Curran, 2004). A qualidade do modelo foi acessada por meio do CFI (Comparative Fit Index), TLI (Tucker-Lewis Index), RMSEA (Root Mean Square Error of Approximation) e SRMR (Standardized Root Mean Square Residual). Valores de RMSEA e SRMR menores que 0,05 indicam bom ajuste, enquanto valores menores que 0,08 indicam ajuste aceitável. CFI e TLI 
acima de 0,95 sugerem ajuste excelente, já valores acima de 0,90 indicam que a qualidade de ajuste é satisfatória (Hu \& Bentler, 1999).

Análises multigrupos foram realizadas para investigar o nível de invariância da EMSVC em função do sexo dos participantes. Modelos aninhados foram comparados por meio do teste do qui-quadrado, além do teste das diferenças entre o CFI e RMSEA dos modelos de mensuração. Uma mudança de CFI menor que $0,01 \mathrm{e}$ uma mudança de RMSEA menor que 0,015 sugerem a preferência por um modelo mais parcimonioso (Cheung \& Rensvold, 2001). O coeficiente alfa de Cronbach e o ômega de McDonald (Dunn, Baguley \& Brunsden, 2014) foram usados para avaliar a consistência interna das dimensões da escala. Considerando que os itens tenderam a apresentar uma violação significativa da distribuição normal multivariada, os alfas foram calculados a partir das correlações não paramétricas de Spearman. Os coeficientes foram interpretados da seguinte forma: $<0,60=$ inadequados; 0,60 a $0,69=$ fidedignidade marginal; 0,70 a $0,79=$ aceitável; 0,80 a $0,89=$ boa; e 0,90 ou mais = excelente (Hair, Black, Babin \& Anderson, 2014). Por último, o teste das diferenças entre os escores obtidos a partir da soma dos escores brutos das respostas dos participantes em cada dimensão foram analisados por sexo e ano escolar. Normas percentílicas por sexo da amostra do presente estudo são apresentadas. Foram utilizados o software R (versão 3.3.1) e as funções implementadas pelo pacote lavaan (Rosseel, 2012).

\section{Resultados}

A AFC especificada de acordo com o modelo original (Giacomoni \& Hutz, 2008) obteve uma solução que convergiu após 264 iterações, não apresentando ajuste adequado aos dados $(\mathrm{CFI}=0,58$ e RMSEA $=0,11)$. Nessa primeira etapa, 15 itens foram deletados por não apresentarem cargas fatoriais significativas com seus respectivos fatores e por terem uma variância explicada pelo modelo menor do que 0,05 (Amizade: EMSVC36, EMSVC25, EMSVC30; Self Comparado: EMSVC2, EMSVC7,
EMSVC12, EMSVC45; Não Violência: EMSVC8, EMSVC22, EMSVC39; Escola: EMSVC21, EMSVC31; Família: EMSVC46, EMSVC38; Self: EMSVC16). Além disso, três itens com cargas cruzadas (EMSVC26, EMSVC20 e EMSVC49) foram retirados dos fatores que possuíam cargas fatoriais não significativas. A análise do novo modelo convergiu após 85 iterações, o que sugere que a solução fatorial foi encontrada mais facilmente do que a solução original, e os índices de ajuste do novo modelo passaram de inadequados para aceitáveis $(\mathrm{CFI}=0,90$ e RMSEA $=0,06$ ).

Igualmente pôde ser observado um aumento da consistência interna das subescalas de acordo com o alfa de Cronbach e ômega de McDonald, exceto a dimensão Amizade $(\omega=0,74)$ nesse último índice, mas, ainda assim, com consistência considerada satisfatória. $\mathrm{O}$ incremento da consistência interna resultou na mudança de três subescalas (Amizade, Família e Self) de consistência interna marginal para boa, a dimensão Não Violência passou de inadequada para marginal e o Self Comparado mudou de consistência boa para excelente, conforme apresentado na Tabela 1.

Por conseguinte, foi testado o modelo da EMSVC com um fator de segunda ordem. Conforme a Tabela 2 , a especificação de um fator geral de satisfação de vida, composto por outras seis dimensões de primeira ordem, resultou no decréscimo do ajuste do modelo (CFI $=0,83$ e RMSEA $=0,08)$. Tal piora no modelo se deve à baixa correlação do fator Não Violência com os outros domínios da EMSVC. Assim, uma análise com um fator de segunda ordem desconsiderando os itens de Não Violência (EMSVC18, EMSVC34, EMSVC47) foi testado. Os índices de ajustes foram satisfatórios $(\mathrm{CFI}=0,90$ e RMSEA $=0,06)$, sugerindo que, para a obtenção de um escore geral de satisfação de vida, as subescalas de Amizade, Escola, Família, Selfe Self Comparado sejam utilizadas para computo do escore composto. Os índices de ajuste do modelo revisado com 35 itens, o modelo refinado de 32 itens com fator de segunda ordem e o modelo de invariância por sexo são apresentados na Tabela 2.

Tabela 1

Fidedignidade EMSVC (Giacomoni \& Hutz, 2008) e EMSVC Revisada

\begin{tabular}{|c|c|c|c|c|c|c|c|c|c|c|c|c|c|c|}
\hline \multirow[b]{2}{*}{ Modelo } & \multicolumn{2}{|c|}{ Amizade } & \multicolumn{2}{|c|}{ Escola } & \multicolumn{2}{|c|}{ Família } & \multicolumn{2}{|c|}{$\begin{array}{c}\text { Não } \\
\text { violência }\end{array}$} & \multicolumn{2}{|c|}{ Self } & \multicolumn{2}{|c|}{$\begin{array}{c}\text { Self } \\
\text { Comparado }\end{array}$} & \multicolumn{2}{|c|}{$\begin{array}{l}\text { Satisfação } \\
\text { de Vida }\end{array}$} \\
\hline & $\alpha$ & $\omega$ & $\alpha$ & $\omega$ & $\alpha$ & $\omega$ & $\alpha$ & $\omega$ & $\alpha$ & $\omega$ & $\alpha$ & $\omega$ & $\alpha$ & $\omega$ \\
\hline Giacomoni \& Hutz (2008) & 0.63 & 0.93 & 0.64 & 0.44 & 0.65 & 0.64 & 0.58 & 0.46 & 0.69 & 0.61 & 0.75 & 0.72 & 0.84 & 0.84 \\
\hline EMSVC Revisada & 0.74 & 0.74 & 0.66 & 0.66 & 0.74 & 0.73 & - & - & 0.71 & 0.70 & 0.84 & 0.84 & 0.88 & 0.90 \\
\hline
\end{tabular}

A análise multigrupo indicou que a EMSVC possui uma forte invariância em função do sexo, em que a restrição da estrutura fatorial da escala, das cargas fatoriais e dos interceptos dos itens nos fatores 
igualmente para os dois grupos indicaram mudanças não significativas nos índices de ajuste (somente o índice TLI caiu para 0,89). Tal propriedade sugere que a escala não apresenta funcionamento diferencial de acordo com o sexo dos participantes (Beaujean, 2014). As propriedades psicométricas do modelo revisado final da EMSVC com 32 itens podem ser visualizadas na Tabela 3.

Tabela 2

Índices de Ajuste dos Modelos

\begin{tabular}{|c|c|c|c|c|c|}
\hline Modelo Confirmatório & $\chi^{2}(g l)$ & CFI & TLI & SRMR & RMSEA \\
\hline Giacomoni \& Hutz (2008) & $18202,84(1153)$ & 0,58 & 0,55 & 0,11 & 0,11 \\
\hline Modelo revisado 35 itens & $2863,45(545)$ & 0,90 & 0,90 & 0,06 & 0,06 \\
\hline Modelo 35 itens Fator de $2^{\text {a }}$ Ordem & $4491,40(552)$ & 0,83 & 0,82 & 0,08 & 0,08 \\
\hline Modelo Fator $2^{a}$ Ordem sem itens de Não-Violência & $2414.59(459)$ & 0,90 & 0,89 & 0,06 & 0,07 \\
\hline Multi-Grupo & $3590,04(1148)$ & 0,90 & 0,89 & 0,06 & 0,07 \\
\hline
\end{tabular}

Nota. CFI=Comparative Fit Index (valor aceitável >0,90), TLI=Tucker-Lewis Index (valor aceitável >0,90), RMSEA=Root Mean Square Error of Approximation (valor aceitável <0,08), SRMR=Standardized Root Mean Square Residual (valor aceitável <0,08).

Tabela 3

Modelo Revisado da EMSVC com Fator Geral: Cargas Fatoriais Padronizadas na Análise Fatorial Confirmatória e Correlações entre Fatores

\begin{tabular}{|c|c|c|c|c|c|}
\hline Itens & Amizade & Escola & Família & Self & Self Comparado \\
\hline EMSVC42 & 0,60 & & & & \\
\hline EMSVC14 & 0,60 & & & & \\
\hline EMSVC48 & 0,56 & & & & \\
\hline EMSVC10 & 0,56 & & & & \\
\hline EMSVC26 & 0,53 & & & & \\
\hline EMSVC4 & 0,45 & & & & \\
\hline EMSVC20 & 0,42 & & & & \\
\hline EMSVC49 & & 0,71 & & & \\
\hline EMSVC43 & & 0,58 & & & \\
\hline EMSVC15 & & 0,54 & & & \\
\hline EMSVC5 & & 0,46 & & & \\
\hline EMSVC29 & & & 0,57 & & \\
\hline EMSVC50 & & & 0,55 & & \\
\hline EMSVC19 & & & 0,54 & & \\
\hline EMSVC41 & & & 0,52 & & \\
\hline EMSVC35 & & & 0,50 & & \\
\hline EMSVC24 & & & 0,47 & & \\
\hline EMSVC3 & & & 0,44 & & \\
\hline EMSVC9 & & & 0,42 & & \\
\hline EMSVC13 & & & 0,37 & & \\
\hline EMSVC11 & & & & 0,60 & \\
\hline EMSVC40 & & & & 0,51 & \\
\hline EMSVC1 & & & & 0,50 & \\
\hline EMSVC6 & & & & 0,50 & \\
\hline EMSVC37 & & & & 0,46 & \\
\hline EMSVC44 & & & & 0,43 & \\
\hline EMSVC32 & & & & 0,42 & \\
\hline EMSVC27 & & & & 0,42 & \\
\hline EMSVC33 & & & & & 0,78 \\
\hline EMSVC28 & & & & & 0,78 \\
\hline EMSVC23 & & & & & 0,75 \\
\hline EMSVC17 & & & & & 0,70 \\
\hline
\end{tabular}


Tabela 3 (continuação)

Modelo Revisado da EMSVC com Fator Geral: Cargas Fatoriais Padronizadas na Análise Fatorial Confirmatória e Correlações entre Fatores

\begin{tabular}{lccccc}
\hline \multicolumn{1}{c}{ Itens } & Amizade & Escola & Família & Self & Self Comparado \\
\hline Satisfação de Vida & 0,99 & 0,83 & 0,89 & 0,96 & 0,16 \\
& & & & & \\
Correlação entre fatores & - & & & & \\
Amizade & $0,58^{* *}$ & - & & \\
Escola & $0,65^{* *}$ & $0,55^{* *}$ & - & & \\
Família & $0,75^{* *}$ & $0,55^{* *}$ & $0,61^{* *}$ & - & \\
Self & $0,21^{*}$ & $0,14^{*}$ & 0,05 & $0,31^{* *}$ & - \\
Self Comparado & &
\end{tabular}

Nota. ${ }^{*} p<0,01 ;{ }^{* *} p<0,001$

A partir do modelo de 32 itens da EMSVC, os escores fatoriais foram computados por meio da soma dos escores brutos dos itens em cada dimensão. Em relação ao sexo, foram encontradas diferenças significativas no teste Kruskal-Wallis para as dimensões: Amizade $(H[1]=4,97$, $p<0,05)$, Escola $(H[1]=5,70, \mathrm{p}<0,05)$, Família $(\mathrm{H}$ $[1]=4,11, p<0,05)$ e Self $(H[1]=5,53 ; 161=p<0,05)$. Também foram encontradas diferenças significativas entre meninos e meninas em relação à satisfação de vida geral $(H=4,97, p<0,05)$. Diante disso, são apresentadas normas das dimensões em função do sexo na Tabela 4.

Em relação ao ano escolar, foram encontradas diferenças significativas em todas as dimensões. As médias dos escores da EMSVC em função do ano escolar são apresentadas na Tabela 5 .

Os resultados encontrados, apesar de significativos, principalmente em relação ao sexto ano apresentaram pequeno tamanho de efeito. A distribuição percentílica em função dos escores brutos computados pode ser observada na Tabela 5 .

Tabela 4

Normas por Sexo da Escala Multidimensional de Satisfação de Vida em Crianças

\begin{tabular}{|c|c|c|c|c|c|c|c|c|c|c|c|c|}
\hline \multirow{2}{*}{ Percentil } & \multicolumn{2}{|c|}{ Amizade } & \multicolumn{2}{|c|}{ Escola } & \multicolumn{2}{|c|}{ Família } & \multicolumn{2}{|c|}{ Self } & \multicolumn{2}{|c|}{ Self Comparado } & \multicolumn{2}{|c|}{ Satisfação de Vida } \\
\hline & $\mathrm{F}$ & $\mathrm{M}$ & $\mathrm{F}$ & $\mathrm{M}$ & $\mathrm{F}$ & $\mathrm{M}$ & $\mathrm{F}$ & $\mathrm{M}$ & $\mathrm{F}$ & $\mathrm{M}$ & $\mathrm{F}$ & $\mathrm{M}$ \\
\hline 0 & 11 & 13 & 4 & 4 & 14 & 16 & 15 & 13 & 4 & 4 & 76 & 68 \\
\hline 5 & 22 & 21 & 11 & 11 & 28 & 29 & 24 & 22 & 5 & 6 & 104 & 97 \\
\hline 10 & 24 & 23 & 12 & 12 & 32 & 31 & 27 & 25 & 6 & 8 & 112 & 107 \\
\hline 15 & 26 & 24 & 13 & 13 & 34 & 33 & 28 & 27 & 8 & 9 & 117 & 113 \\
\hline 20 & 27 & 25 & 14 & 14 & 35 & 34 & 29 & 28 & 9 & 11 & 121 & 118 \\
\hline 25 & 28 & 26 & 15 & 14 & 36 & 35 & 30 & 29 & 10 & 11 & 124 & 122 \\
\hline 30 & 28 & 27 & 16 & 15 & 37 & 36 & 31 & 30 & 12 & 12 & 127 & 125 \\
\hline 35 & 29 & 28 & 16 & 16 & 38 & 37 & 32 & 31 & 13 & 13 & 129 & 127 \\
\hline 40 & 29 & 28 & 17 & 16 & 39 & 38 & 33 & 32 & 14 & 14 & 131 & 130 \\
\hline 45 & 30 & 29 & 17 & 16 & 39 & 39 & 33 & 32 & 15 & 15 & 132 & 131 \\
\hline 50 & 30 & 29 & 18 & 17 & 40 & 39 & 34 & 33 & 16 & 16 & 135 & 132 \\
\hline 55 & 31 & 30 & 18 & 17 & 41 & 40 & 34 & 34 & 16 & 17 & 137 & 134 \\
\hline 60 & 31 & 31 & 18 & 18 & 41 & 41 & 35 & 34 & 17 & 17 & 139 & 136 \\
\hline 65 & 32 & 31 & 19 & 18 & 42 & 41 & 35 & 35 & 18 & 18 & 140 & 139 \\
\hline 70 & 32 & 32 & 19 & 19 & 42 & 42 & 36 & 35 & 18 & 18 & 142 & 140 \\
\hline 75 & 33 & 32 & 19 & 19 & 43 & 43 & 36 & 36 & 19 & 18 & 143 & 142 \\
\hline 80 & 34 & 33 & 20 & 20 & 44 & 43 & 37 & 37 & 19 & 19 & 146 & 145 \\
\hline 85 & 34 & 34 & 20 & 20 & 44 & 44 & 38 & 37 & 20 & 20 & 148 & 147 \\
\hline 90 & 35 & 35 & 20 & 20 & 45 & 44 & 39 & 38 & 20 & 20 & 152 & 151 \\
\hline 95 & 35 & 35 & 20 & 20 & 45 & 45 & 40 & 40 & 20 & 20 & 156 & 155 \\
\hline 100 & 35 & 35 & 20 & 20 & 45 & 45 & 40 & 40 & 20 & 20 & 160 & 160 \\
\hline
\end{tabular}

Nota. $\mathrm{F}=$ feminino; $\mathrm{M}=$ masculino 
Tabela 5

Percentis dos Escores da EMSVC em Função do Ano Escolar

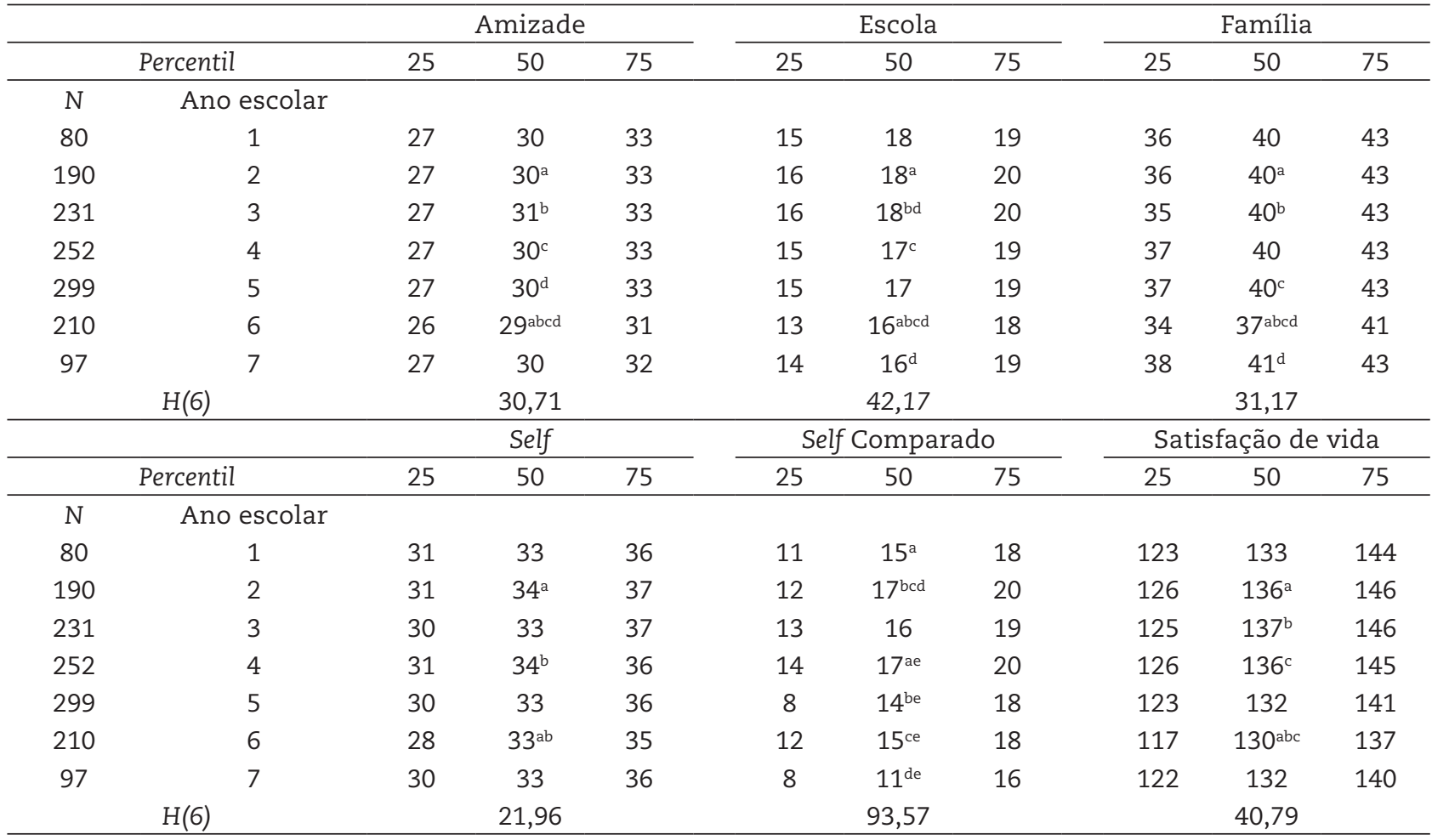

Nota. As letras a até $l$ em sobrescrito foram usadas para identificação das medianas das subescalas que diferiram significativamente em função do ano escolar no teste Kruskal-Wallis. Em cada coluna, duas letras iguais indicam médias significativamente diferentes na análise post-hoc Bonferroni. ${ }^{*} p<0,05$

\section{Discussão}

O objetivo deste estudo foi revisar as propriedades psicométricas da EMSVC, investigar a sua estrutura interna e criar normas para a utilização do instrumento. Conforme os resultados, os índices de ajuste do novo modelo passaram de inadequados para aceitáveis e houve um incremento na consistência interna das subescalas que compõem o instrumento.

Após o refinamento por meio da AFC, o modelo final composto por 35 itens da EMSVC permaneceu com as seis dimensões orginalmente propostas (Giacomoni \& Hutz, 2008), distribuídas da seguinte forma: Self (oito itens), Self Comparado (quatro itens), Não Violência (dois itens), Família (nove itens), Amizade (sete itens) e Escola (quatro itens). Todas as cargas fatoriais carregaram positiva e significamente nos seus respectivos fatores. Entretanto, na especificação de um fator geral de satisfação de vida, o fator Não Violência demonstrou baixa fidedignidade e baixa correlação com os outros domínios. Tal resultado também foi observado no estudo original (Giacomoni \& Hutz, 2008), no qual os autores justificaram esse valor mais baixo $(\alpha=0,66)$ devido ao pequeno número de itens dessa subescala $(n=4)$. Assim, é possível identificar um funcionamento deficitário da dimensão desde a versão inicial do instrumento.
Tendo em vista que o fator Self Comparado ficou composto por quatro itens após esta revisão e apresentou índices satisfatórios de fidedignidade, a justificativa da baixa quantidade de itens do fator Não Violência parece não se sustentar.

Alguns estudos apresentaram a EMSVC em uma versão reduzida (Bandeira, 2014; Pedro, 2017) sem incluir os itens da dimensão Não Violência, embora não tenham especificado os procedimentos que justifiquem a redução. Em um estudo recente (Cassoni et al., 2017) os autores sugeriram a eliminação de dois itens da dimensão Não Violência devido à baixa fidedignidade $(\alpha=0,50)$ e justificaram que provavelmente o fator seja menos confiável para tirar conclusões sobre a satisfação de vida das crianças por causa da desejabilidade social. Nessa direção, propõe-se, para compor o escore geral da escala, apenas a utilização das subescalas Amizade, Escola, Família, Self e Self Comparado, totalizando 32 itens.

A respeito das intercorrelações dos fatores, identificou-se uma alta correlação entre as dimensões Amizade e $\operatorname{Self}(r=0,75, p<0,001)$. Hipotetizou-se que a magnitude dessa associação poderia sugerir que esses dois domínios comporiam uma única dimensão, entretanto, apesar da análise fatorial considerando uma única dimensão de Amizade/Self ter indicado índices de ajuste satisfatórios, o conteúdo dos itens se refere a aspectos 
distintos do construto satisfação de vida. Dessa forma, optou-se pela manutenção da estrutura com cinco dimensões devido à proposta teórica que embasa o modelo de mensuração.

Diferenças entre os anos escolares em todas as dimensões de satisfação de vida foram observadas no presente estudo. De modo específico, nota-se uma diminuição dos níveis a partir do $5^{\circ}$ ano do ensino fundamental. No contexto da educação brasileira, no $5^{\circ}$ ano ocorre um importante aumento de demandas relacionadas à educação formal, entre elas, a passagem da unidocência para a pluridocência e a elevação das expectativas sobre o desempenho acadêmico, o que gera maior preocupação entre os alunos (Pereira \& Silveira, 2017), podendo este ser um fator que influencia no bem-estar subjetivo a partir deste período. Além disso, no artigo de desenvolvimento da EMSVC (Giacomoni \& Hutz, 2008), foi identificado que crianças mais novas apresentavam maiores níveis de satisfação de vida nos domínios Self, Família, Amizade e Escola, ocorrendo um decréscimo com o passar dos anos. Assim, é possível que aspectos do desenvolvimento humano também tenham influenciado nas diferenças encontradas, à medida que alunos que frequentam o $5^{\circ}$ ano do ensino fundamental, em sua maioria, estão passando por mudanças físicas e comportamentais típicas da fase de transição para a adolescência (Papalia \& Feldman, 2013).

Foi identificada ainda diferença significativa entre meninos e meninas para a satisfação de vida geral e nas dimensões Amizade, Escola, Família e Self. No estudo original (Giacomoni \& Hutz, 2008), tais diferenças em relação ao sexo não foram verificadas. Ainda que predominem na literatura achados que não evidenciam discrepâncias entre sexos, alguns estudos sugerem resultados distintos para meninos e meninas em áreas específicas e mais relacionados com aspectos que envolvem relações interpessoais. Chui e Wong (2016) identificaram em uma amostra de adolescentes que as meninas tenderam a apresentar níveis mais altos de satisfação de vida relacionados à escola, embora não fossem encontradas diferenças no nível de satisfação de vida geral para ambos os sexos. Na mesma direção, Piko e Hamvai (2010) indicaram que, para as meninas, a área das amizades contribuiria de maneira mais marcante para seus níveis de satisfação de vida, do que para meninos. Tais resultados podem apontar direções para futuras investigações que melhor explorem as possíveis diferenças entre os domínios conforme o sexo. Entretanto, nesse momento, sugere-se interpretar as discrepâncias evidenciadas com cautela uma vez que os resultados observados no presente estudo possuem pequeno tamanho de efeito. A respeito das diferenças entre sexos em outros aspectos, o estudo de Balazs, Miklosi, Toro e Nagy-Varga (2016) salienta a necessidade de considerar o efeito moderador do sexo do participante na relação entre qualidade de vida e comorbidade psicopatológica em crianças com e sem deficiência em leitura. Segundo os autores a interação também é evidenciada ao serem modelados os efeitos de idade das crianças. Ressalta-se que o efeito de interação foi observado em crianças do grupo controle. Similarmente, Schonert-Reichl et al. (2015) apontaram a necessidade de controlar o efeito de sexo na implementação de uma intervenção baseada em mindfullness para aprimoramento de desenvolvimento socioemocional e cognitivo de estudantes do ensino fundamental.

Apesar das contribuições deste estudo, cabe apontar uma limitação no que diz respeito à composição da amostra com crianças provenientes de apenas um estado do Brasil. Além disso, por ser uma compilação de dados de pesquisas anteriores, não foi possível acessar o tipo de escola de alguns participantes, uma variável que é considerada importante devido às suas diferenças econômicas e contextuais (Menezes et al., 2016). Ademais, é importante realizar outros estudos para a elaboração de normas da EMSVC com amostras de outros estados que abranjam a realidade socioeconômica e cultural do país.

Este estudo contribui para o aprimoramento da escala e fornece as normas por sexo e ano escolar para uso clínico e de pesquisa da EMSVC, um instrumento útil para a investigação de um dos componentes do bem-estar em crianças e pré-adolescentes. Salienta-se a importância de investir em instrumentos que avaliem construtos positivos, os quais são importantes para a utilização em avaliação de intervenção no contexto clínico ou escolar. O leitor interessado pode acessar a EMSVC na versão reduzida consultando o site do Núcleo de Estudos em Psicologia Positiva (NEPP) da UFRGS (http://www.ufrgs.br/nepp/).

\section{Referências}

Bandeira, C. M. (2014). Desenvolvimento positivo infantil: As relações entre otimismo, afetos e satisfação de vida (Tese de doutorado não publicada). Universidade Federal do Rio Grande do Sul.

Balazs, J., Miklosi, M., Toro, K. T., \& Nagy-varga, D. (2016). Reading disability and quality of life based on both self- and parent-reports: Importance of gender differences. Frontiers in Psychology, 7(December), 1-9. doi: 10.3389/fpsyg.2016.01942

Beaujean, A. A. (2014). Latent variable modeling using $R$. Latent variable modeling using R: A step-by-step guide. New York: Routledge. doi: $10.4324 / 9781315869780$ 
Cassoni, C., Marturano, E. M., Coimbra, S., \& Fontaine, A. M. (2017). A validation study of the Multidimensional Life Satisfaction Scale for Children. Psicologia: Reflexão e Crítica, 30(14). doi: 10.1186/s41155-017-0068-6

Cheung, G. W., \& Rensvold, R. B. (2001). The effects of model parsimony and sampling error on the fit of structural equation models. Organizational Research Methods, 4(3), 236-264. doi: 10.1177/109442810143004

Chui, W. H., \& Wong, M. Y. H. (2016). Gender differences in happiness and life satisfaction among adolescents in Hong Kong: Relationships and self-concept. Social Indicators Research, 125, 1035-1051. doi: 10.1007/s11205-015-0867-z

Dell'Aglio, D. D., \& Siqueira, A. C. (2010). Preditores de satisfação de vida de jovens em situação de vulnerabilidade no sul do Brasil. Psicodebate: Psicología, Cultura y Sociedad, 10, 213-130. doi: 10.18682/pd.v10i0.397

Diener, E. (1984). Subjective well-being. Psychological Bulletin, 95(3), 542-575. doi: 10.1037/0033-2909.95.3.542

Diener, E, Heintzelman, S. J., Kushlev, K., Tay, L., Wirtz, D., Lutes, L. D., \& Oishi, S. (2017). Findings all psychologists should know from the new science on subjective well-being. Canadian Psychology, 58(2), 87-104. doi: 10.1037/cap0000063

Dunn, T. J., Baguley, T., \& Brunsden, V. (2014). From alpha to omega: A practical solution to the pervasive problem of internal consistency estimation. British Journal of Psychology, 105(3), 399-412. doi: 10.1111/bjop.12046

Flora, D. B., \& Curran, P. J. (2004). An empirical evaluation of alternative methods of estimation for confirmatory factor analysis with ordinal data. Psychological Methods, 9(4), 466-491. doi: 10.1037/1082-989X.9.4.466.An

França-Freitas, M. L. P., Del Prette, A., \& Del Prette, Z. (2017). Habilidades sociais e bem-estar subjetivo de crianças dotadas e talentosas. Psico-USF, 22(1), 1-12. doi: 10.1590/1413-82712017220101

Giacomoni, C. H. (2002). Bem-estar subjetivo infantil: Conceito de felicidade e construção de instrumentos para avaliação (Tese de doutorado). Curso de Pós-Graduação em Psicologia do Desenvolvimento. Universidade Federal do Rio Grande do Sul: Porto Alegre.

Giacomoni, C. H., \& Hutz, C. S. (2008). Escala multidimensional de satisfação de vida para crianças: Estudos de construção e validação. Estudos de Psicologia (Campinas), 25(1), 25-35. doi: 10.1590/S0103-166X2008000100003

Gilman, R., \& Huebner, E. S. (2006). Characteristics of adolescents who report very high life satisfaction. Journal of Youth and Adolescence, 35(3), 311-319. doi: 10.1007/s10964-006-9036-7

Gonçalves, S. C. O. (2013). Educação Positiva na promoção do bem-estar em crianças do $4^{\circ}$ ano de Escolaridade (Dissertação de mestrado). Universidade do Algave, Faro, Portugal.

Hair, J. F., Black, W. C., Babin, B. J., \& Anderson, R. E. (2014). Multivariate Data Analysis (7th ed.). Harlow, UK: Pearson Education Limited.

Hu, L., \& Bentler, P. M. (1999). Cutoff criteria for fit indexes in covariance structure analysis: Conventional criteria versus new alternatives. Structural Equation Modeling: A Multidisciplinary Journal, 6(1), 1-55. doi: 10.1080/10705519909540118

Huebner, E. (1994). Preliminary development and validation of a multidimensional life satisfaction scale for children. Psychological Assessment, 6(2), 149-158. doi: 10.1037/1040-3590.6.2.149

Martins, D. I. B. (2012). Depressão e satisfação de vida em crianças e adolescentes institucionalizados e não institucionalizados: Estudo comparativo (Dissertação de mestrado). Universidade da Beira Interior, Covilhã, Portugal.

Menezes, I. G., Duran, V. R., Mendonça Filho, E. J., Veloso, T. J., Sarmento, S. M. S., Paget, C. L., \& Ruggeri, K. (2016). Policy implications of achievement testing using multilevel models: The case of Brazilian elementary schools. Frontiers in Psychology, 7(1727). doi: 10.3389/ fpsyg.2016.01727

Papalia, D. E., \& Feldman, R. D. (2013). Desenvolvimento Humano (12. ed). Porto Alegre, RS: Artmed.

Pedro, V. C. S. M. D. (2017). A Educação Positiva como base de um modelo de intervenção psicológica com alunos do ensino básico (Dissertação de mestrado). Universidade do Algarve, Faro, Portugal.

Pereira, L. O, A., \& Silveira, L. M. O. B. (2017). Percepção das professoras sobre seus alunos do $5^{\circ}$ ano. Psicologia da Educação, 45, 77-86. doi: $10.5935 / 2175-3520.20170019$

Piko, B. F., \& Hamvai, C. (2010). Parent, school and peer-related correlates of adolescents' life satisfaction. Children and Youth Services Review, 32(10), 1479-1482. doi: 10.1016/j.childyouth.2010.07.007

Pincolini, A. M., F., \& Hutz, C. S. (2012). Bem-estar subjetivo em famílias com histórico de abuso sexual intrafamiliar. Gerais: Revista Interinstitucional de Psicologia, 5(1), 3-22. Recuperado de http://pepsic.bvsalud.org/scielo.php?script=sci_arttext\&pid=S1983$82202012000100002 \& \operatorname{lng}=\mathrm{pt} \& \mathrm{t} \operatorname{lng}=\mathrm{pt}$

Pires, J. G., Nunes, M. F. O., \& Nunes, C. H. S. S. (2015). Instrumentos baseados em psicologia positiva no Brasil: Uma revisão sistemática. Psico-USF, 20(2), 267-295. doi: 10.1590/1413-82712015200209

Poletto, M., \& Koller, S. H. (2011). Bem-estar subjetivo em crianças e adolescentes em situação de vulnerabilidade social. Psicologia: Reflexão e Crítica, 24(3), 476-484. doi: 10.1590/S0102-79722011000300008

Pureza, J. R., Kuhn, C. H., Castro, E. K., \& Lisboa, C. S. M. (2012). Psicologia positiva no Brasil: Uma revisão sistemática da literatura. Revista Brasileira de Terapias Cognitivas, 8(2), 109-117. doi: 10.5935/1808-5687.20120016

Reppold, C., Gurgel, L. G., \& Schiavon, C. C. (2015). Pesquisa em psicologia positiva: Uma revisão sistemática da literatura. Psico-USF, 20(2), 275-285. doi: 10.1590/1413-82712015200208

Rosseel, Y (2012). Lavaan: An R package for structural equation modeling. Journal of Statistical Software, 48(2), 1-36. Recuperado de http:// www.jstatsoft.org/v48/i02/

Schonert-Reichl, K. A., Oberle, E., Lawlor, M. S., Abbott, D., Thomson, K., Oberlander, T. F., \& Diamond, A. (2015). enhancing cognitive and social - emotional development through a simple-to-administer mindfulness-based school program for elementary school children: A randomized controlled trial. Developmental Psychology, 51(1), 52-66. doi: 10.1037/a0038454

Suldo, S. M., Huebner, E. S., Friedrich, A. A., \& Gilman, R. (2009). Life satisfaction. Em R. Gilman, E. S. Huebner, \& M. Furlong (Eds.). Handbook of positive psychology in the schools (pp. 27-35). New York: Routledge 
Suldo, S. M., Frank, M. J., Chappel, A. M., Albers, M. M., \& Bateman, L. P. (2014). American high school students' perceptions of determinants of life satisfaction. Social Indicators Research, 118(2), 485-514. doi: 10.1007/s11205-013-0436-2

\section{Sobre os autores}

Cyntia Mendes de Oliveira é psicóloga, mestre em Psicologia, bolsista CAPES do doutorado em Psicologia pela Universidade Federal do Rio Grande do Sul e membro do Núcleo de Estudos em Psicologia Positiva.

Euclides José de Mendonça Filho é psicólogo, mestre em Psicologia, bolsista CAPES do doutorado em Psicologia pela UFRGS e membro do Grupo de Estudos, Aplicação e Pesquisa em Avaliação Psicológica.

Aline Riboli Marasca é psicóloga, mestranda em Psicologia, bolsista CNPQ do mestrado em Psicologia pela UFRGS e membro do Grupo de Estudos, Aplicação e Pesquisa em Avaliação Psicológica.

Denise Ruschel Bandeira é doutora em Psicologia (UFRGS), professora do Programa de Pós-Graduação em Psicologia da UFRGS e coordenadora do Grupo de Estudos, Aplicação e Pesquisa em Avaliação Psicológica.

Claudia Hofheinz Giacomoni é doutora em Psicologia (UFRGS), professora do Departamento de Psicologia do Desenvolvimento e da Personalidade e do Programa de Pós-graduação em Psicologia da UFRGS e coordenadora do Núcleo de Estudos em Psicologia Positiva. 